\title{
Variations in technology
}

Kenneth L. Mattox, MD

From the Department of Surgery, Baylor College of Medicine, Houston, Tex.

Received for publication Aug 23, 2000; accepted for publication Aug 24, 2000.

Address for reprints: Kenneth L. Mattox, MD, Department of Surgery, Baylor College of Medicine, One Baylor Plaza, Houston, TX 77030.

J Thorac Cardiovasc Surg 2003;125:S66-7 Originally published in J Thorac Cardiovasc Surg 2000;120:1102-3.

Copyright () 2003 by The American Association for Thoracic Surgery

0022-5223/2003 $\$ 30.00+0$

doi: $10.1067 / \mathrm{mtc} .2003 .229$

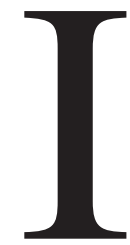

n this issue of the Journal [J Thorac Cardiovasc Surg 2000;120:1104-11], Downing and associates from The R. Adams Cowley Shock Trauma Center of the University of Maryland School of Medicine continue their long-term contributions to the treatment of aortic injury. They report on their 66month experience with 50 patients in whom heparinless right atrial (via femoral vein cannulation) to femoral artery pump bypass was used. The etiology, pathophysiology, suspicion, diagnosis, timing, and type of treatment, as well as prevention of complications and death after acute blunt injury to the thoracic aorta, have been the focus of more than 3000 articles in the literature. Traditional femoral vein-femoral artery bypass with full-body heparinization was extensively used during the late 1960s through the mid-1980s. Other significant reports of heparinless technology using a centrifugal pump with cannulation in other sites are in the literature. In the acutely injured patient, it is appropriate for the surgeon to avoid total heparinization and its resultant coagulopathy. The report by Downing and associates is significant, in that the venous cannula is inserted via the femoral vein into the right atrium, either at the time of the thoracic aortogram or immediately after induction of anesthesia. In the discussion, the authors continue to reidentify some of the many continuing controversies relating to acute aortic injury.

Throughout the years, a number of intraoperative approaches, including simple clamp repair, Gott shunts, traditional cardiopulmonary bypass, and the use of centrifugal pumps with and without heparin, have been reported by various authors. Excellent and almost identical results have been reported by selected authors using each technique, many reports of which are referenced in this report. A surgeon operating on a patient with an aortic injury must be familiar with each of the approaches and might need to use one or another, depending on the extent of the injury. Significant complications and death have also been reported after use of each of the techniques. None is clearly superior. No technique should be summarily championed, and none should be condemned outright.

The Maryland Institute for Emergency Medical Services System (MIEMSS) group, like others reporting large series, cites a range in clamp times (14-70 minutes). Reports of longer clamp times (or even permanent exclusion of the aorta) without paraplegia exist, and paraplegia has been reported after clamp times of less than 30 minutes. The authors' citation of "crossclamp times greater than 30 minutes" is oft repeated in the literature and not specifically supported by the data in the report by Downing and associates, nor in all of their cited literature. This group is to be commended for their results using their approach to distal perfusion; however, similar results were recently reported by Sweeny and associates (reference 15 in their article) using simple clamp/repair without distal perfusion. A surgeon should not feel compelled to "rush" an operation just to finish within 30 minutes. Furthermore, as was discovered after years of reliance on the passive shunt (whose flow rates were unpredictable and undependable), a surgeon should not feel a sense of added security just because an active shunt is used. I would suggest that the oft-repeated recommendation to keep the crossclamp time less than 30 minutes not only is not supported by evidence-based reports, but has tremendous medical/legal implications. It is entirely possible that future prospective evaluations will show that time is not an independent variable in the appearance of paraplegia after injury repair. Many other factors exist that contribute to this dreaded complication of thoracic aortic trauma and surgery.

It is appropriate that surgeons continue to apply emerging technology and 
information in an effort to improve the care of injured patients. Among selected patients, a number of technologies require close scrutiny for future appropriate application to aortic trauma: these include purposeful delay in operation, transesophageal echocardiography, 3-dimensional reconstruction of advanced enhanced spiral computed tomographic imaging, draining of cerebral spinal fluid to prevent spinal compartment syndrome, enhanced angiography to detect aortic arch anomalies, and endovascular stented graft repair of chronic posttraumatic pseudoaneurysms. The authors' contribution of a percutaneously inserted venous drainage catheter connected to an in-line centrifugal pump and heat exchanger before direct return via an aortic cannula is one such technology which has been advantageous in their treatment schema. 